\title{
A preliminary qualitative study of general practitioners' views on malnutrition management and oral nutritional supplementation prescription in the community
}

\author{
Patricia Domínguez Castro $^{1,2}$, Sharon Kennelly ${ }^{3}$, Barbara Clyne ${ }^{4}$, Gerard Bury ${ }^{5}$, \\ Catriona Bradley ${ }^{6}$, Karen Finnigan ${ }^{7}$, Laura McCullagh ${ }^{7}$, Michael Barry ${ }^{7}$, Celine Murrin ${ }^{1,2}$, \\ Carla Perrotta $^{1,2}$, Katherine Loayza Villaroel ${ }^{1,2}$, Ciara Reynolds ${ }^{1,2}$, Eileen Gibney ${ }^{2,8}$ and \\ Clare Corish ${ }^{1,2}$ \\ ${ }^{1}$ School of Public Health, Physiotherapy and Sports Science, University College Dublin, Dublin, Ireland, \\ ${ }^{2}$ Institute of Food and Health, University College Dublin, Dublin, Ireland, \\ ${ }^{3}$ National Primary Care Division, Community Funded Schemes Service Improvement, Dublin, Ireland, \\ ${ }^{4}$ HRB Centre for Primary Care Research, Department of General Practice, Royal College of Surgeons in Ireland, \\ Dublin, Ireland, \\ ${ }^{5}$ School of Medicine, University College Dublin, Dublin, Ireland, \\ ${ }^{6}$ Royal College of Surgeons in Ireland, Dublin, Ireland, \\ ${ }^{7}$ Department of Pharmacology and Therapeutics, Trinity Centre for Health Sciences, St James's Hospital, Dublin, \\ Ireland and \\ ${ }^{8}$ School of Agriculture and Food Science, University College Dublin, Dublin, Dublin, Ireland
}

\begin{abstract}
Malnutrition, arising from a deficiency of energy and protein intake, occurs commonly among community-dwelling individuals. Oral nutritional supplements (ONS) are an effective method of managing malnutrition if prescribed for patients who are malnourished or at risk of malnutrition. However, previous research indicates that inappropriate prescribing of ONS occurs frequently in the community setting (30-70\% prevalence). Moreover, it has been previously shown that the management of patients 'at risk' of malnutrition in the primary care/community setting in Ireland is sub-optimal, with low awareness of the condition and its management among nondietetic health care professionals (HCPs). In Ireland, general practitioners (GPs) are the main prescribers of ONS in primary care. They are also on many occasions the first point of contact for individuals in the community who are malnourished or at risk of malnutrition. Therefore, exploring their experiences and perceptions can provide insight into the complexities of managing malnutrition and ONS prescribing in the community. The aim of this qualitative study was to explore GPs' experiences and opinions on the management of malnutrition and the prescription of ONS in the primary care/community setting in Ireland. Semi-structured interviews were conducted with nine GPs. The interview explored among others the following domains; Barriers and facilitators in the management of malnutrition, ONS prescribing in the primary care/community setting, and future directions in the management of malnutrition and ONS prescribing. Recorded interviews were transcribed and analysed using inductive thematic analysis. Three main themes were identified; i) Malnutrition a secondary concern, encapsulating the idea that malnutrition is not something that GPs screen for in isolation ii) Resources and support for managing malnutrition are needed in the community iii) Reluctance to prescribe ONS. GPs reported not screening for malnutrition, but viewed it as a consequence of other conditions and social factors. According to the GPs, they should not have sole responsibility for malnutrition management and more dietitians and trained community nurses would be desirable to support them to deal with malnutrition in the community. A preference to avoid supplementation and implement dietary changes first was expressed by GPs. As main prescribers, GPs thought that they should receive further independent trainingon the different ONS available in the market as currently they did not feel confident prescribing a range of them. Awareness of ONS cost and its misuse by certain patients were reported as reasons that made GPs reluctant to prescribe them, potentially leading to under prescribing.
\end{abstract}

\section{Conflict of Interest}

There is no conflict of interest. 